Archives de sciences sociales des religions

176 | octobre-décembre 2016

Bulletin Bibliographique

\title{
Avec Émile Poulat, à la découverte des arcanes de la crise moderniste (1950-1962)
}

Giacomo Losito

\section{OpenEdition}

1 Journals

Édition électronique

URL : http://journals.openedition.org/assr/28277

DOI : $10.4000 /$ assr.28277

ISSN : $1777-5825$

Éditeur

Éditions de l'EHESS

Édition imprimée

Date de publication : 31 décembre 2016

Pagination : 41-54

ISSN : 0335-5985

\section{Référence électronique}

Giacomo Losito, «Avec Émile Poulat, à la découverte des arcanes de la crise moderniste (1950-1962)

", Archives de sciences sociales des religions [En ligne], 176 | octobre-décembre 2016, mis en ligne le 01 janvier 2019, consulté le 04 janvier 2020. URL : http://journals.openedition.org/assr/28277 ; DOI :

10.4000/assr.28277

(C) Archives de sciences sociales des religions 


\section{Giacomo Losito}

\section{Avec Émile Poulat, à la découverte des arcanes de la crise moderniste (1950-1962) ${ }^{1}$}

Émile Poulat est incontestablement le grand spécialiste français de la crise moderniste. Sa thèse de 1962 est une œuvre inaugurale, un travail conduit dans l'intention de formuler une lecture explicative, et donc la plus objective possible, de l'une des crises les plus aigües et aux effets passionnels les plus durables de l'histoire du catholicisme; c'est une œuvre qui a par la suite servi de modèle à des générations d'historiens du catholicisme contemporain, en France comme à l'étranger. Elle fut préparée sur la base d'un travail documentaire approfondi et d'une étude préalable de dossiers ${ }^{2}$ dont le soin sera une marque caractéristique de toute la production d'Émile Poulat, qui ne manquera pas non plus d'offrir par la suite d'autres textes de référence dans le champ des études sur la crise moderniste, notamment son édition de "Sylvain Leblanc»(Henri Bremond), Un clerc qui n'a pas trabi. Alfred Loisy d'après ses Mémoires, en 1972. Poulat a consciemment revendiqué comme un caractère personnel le style " froid ${ }^{3}$ de son approche d'un objet historique qui, à un demi-siècle de distance, restait encore un objet incandescent, à manipuler avec des pincettes :

La crise moderniste évoque un champ de bataille encore miné [...]. Qu'on s'approche, et l'on découvre vite, avec étonnement, que la passion ni la peur ne sont éteintes

1. Nous nous limiterons à l'étude des textes publiés. Ce choix, imposé par les circonstances (dans l'attente que les archives Poulat, désormais déposées à Nantes et actuellement en cours d'inventaire, soient accessibles aux chercheurs), est aussi fidèle à la démarche intellectuelle de notre auteur, qui justifiait la primauté de l'imprimé sur l'inédit comme « celle de la pensée construite et de la parole publique sur l'échange privé, qui n'a pas le même statut intellectuel et social » ("Avant-propos à la $3^{\mathrm{e}}$ édition », Poulat, 1996a : XIII). Nous remercions aussi François Trémolières pour ses conseils et ses encouragements à réaliser cet article.

2. Voir (Houtin, 1960); (Poulat, 1958). Dans la note finale de sa "Préface " à la thèse de 1962 (Poulat, 1996a : 29), Poulat a évoqué sa "curiosité de chercheur " qui l'avait poussé à fouiller dans les archives «de l'Ardèche à la Touraine, de l'Écosse à l'Italie ». Les rapporteurs de la soutenance ont enregistré des précisions pour ce qui concerne les archives à l'étranger : Cambridge, St Andrews, Gênes et Florence (Bédarida, 1963 : 263).

3. "Je n'ai jamais traité que de problèmes brûlants et même explosifs, mais sur le mode froid et professionnel » (Émile Poulat, "Une pratique méditative de l'histoire ", dans Rivista di storia sociale releligiosa, 2002-1, 17-28, repris dans Poulat, 2014 : 67). 
autour de zones qui demeurent interdites, autour de morts qui les hantent, d'inimitiés et de blessures dont le souvenir reste vif, de défiances toujours en éveil, parfois même, il semble que le feu couve encore sous la cendre et que la vigilance s'impose toujours ${ }^{4}$.

Pourtant, il ne s'est jamais laissé enliser dans les pièges d'un positivisme naïf et il a toujours souligné la part qui revient à la subjectivité du savant en tout travail de sciences humaines ${ }^{5}$ : simple précaution de la part d'un esprit bien éveillé à ce problème ${ }^{6}$ contre une accusation qui fut précocement formulée à l'égard de la méthode qu'il avait adoptée ${ }^{7}$ ? Chercher une réponse à cette question nous permettra aussi de faire un peu mieux connaissance avec Poulat et de comprendre pourquoi et comment il s'attacha à la crise moderniste.

En fait, il est notoire que l'étude par Poulat de la crise moderniste constitue une partie - importante - de sa lecture plus globale de l'histoire du catholicisme contemporain $^{8}$ servie par la grille explicative socio-historique du conflit triangulaire entre la démocratie libérale, le socialisme et l'intégralisme catholique, où ce dernier constitue aussi la matrice de différents courants, souvent en féroce compétition entre eux (catholicisme social, démocratie chrétienne, Action catholique, intégrisme et même... modernisme $)^{9}$. La rencontre et les échanges avec les spécialistes de l'histoire religieuse de l'Italie contemporaine, où un rôle prépondérant fut joué par le courant du catholicisme intransigeant, mais aussi avec ceux d'autres terres électives de la chrétienté comme le Québec et le Mexique, ont certainement aidé Poulat dans l'élaboration de ses clés de compréhension d'une réalité historique complexe. Mais nous entendons montrer que la recherche du pionner des études sur la crise moderniste, stimulée et même orientée par son analyse de ses propres péripéties ecclésiales, contenait déjà les prémisses théoriques de ce qui sera formalisé ensuite quant à la nature matricielle de l'intransigeance pour le catholicisme confronté à la démocratie bourgeoise et au socialisme, et cela avant même l'ouverture de son regard au-delà des confins du catholicisme hexagonal. Nous verrons que cette orientation théorique des recherches du jeune

4. «Préface» de 1962 (Poulat, 1996a: 7).

5. Voir notamment (Poulat, 1996a : 16); et « Compréhension historique de l'Église et compréhension ecclésiale de l'histoire ", dans Concilium, 67, septembre 1971, p. 15-27, repris dans (Poulat, 1982 : 273-287) et dans (Poulat, $2014: 121-140$, ici p. 124, 125 et 135).

6. De la connaissance historique d'Henri-Irénée Marrou date de 1954 et, d'ailleurs, le débat sur les conditions subjectives du savoir historique constituait déjà un enjeu considérable de l'affrontement Loisy-Blondel ponctuellement étudié par Poulat dans sa thèse de 1962.

7. Voir (Bédarida, 1963). Le compte rendu de la soutenance de thèse d'Émile Poulat reprend des observations formulées par les membres du jury, Henri Gouhier, Gabriel Le Bras, Henri-Irénée Marrou et Alphonse Dupront. Voir aussi l'introduction de Guido Verucci à la traduction italienne de la thèse de 1962 (Brescia, Morcelliana, p. IX-XXVI), notamment p. XXV-XXVI.

8. L'affirmation suivante est éclairante sur le sens que Poulat a donné à ses études modernistes : «Mon effort a toujours visé [...] à sortir le modernisme de la chronique et de l'éristique où son histoire risquait de s'enliser sans profit pour personne » (Poulat, 1984: 7).

9. Ce sont les thèses directrices exposées dans le diptyque de 1977 : (Poulat, 1977) et (Poulat, 2006a). 
membre du Groupe de sociologie des religions du CNRS lui venait donc non seulement de son engagement intellectuel à l'école de Gabriel Le Bras et d'Ignace Meyerson ${ }^{10}$, mais aussi de sa propre compréhension du dramatique vécu ecclésial dont il avait été l'un des protagonistes ${ }^{11}$ et l'historien : la crise des prêtresouvriers de $1954^{12}$.

\section{***;:}

La récente publication de la thèse de théologie présentée au début de 1950 à l'université allemande de Fribourg (Poulat, 2015) - lorsque Poulat venait d'intégrer la Mission de Paris - a permis de montrer que le jeune clerc (ordonné à Paris en 1945) était déjà bien documenté et tout à fait conscient de l'importance jouée par le modernisme dans l'histoire de la pensée catholique. En particulier, Poulat y montre l'effervescence culturelle et l'essai de renouvellement des études religieuses qui ont caractérisé la fin du pontificat de Léon XIII et celui de Pie X, notamment l'épanouissement de la philosophie de l'action blondélienne au début du renouvellement contemporain de la théologie du désir naturel de Dieu. Toutefois, en 1950, d'après la leçon du cardinal Suhard ${ }^{13}$ et à la suite de Blondel, Poulat n'hésita pas à traiter le modernisme $d^{\prime}$ " illusion » et d'erreur ${ }^{14}$; aussi n'est-ce pas un hasard si "le grand homme de la thèse » ${ }^{15}$ de théologie fribourgeoise est justement Maurice Blondel, alors que, comme on le sait, le personnage clé de la thèse de 1962 sera plutôt le "père du modernisme " ${ }^{16}$, son "personnage éponyme » (Poulat, 1996 : 19) : Alfred Loisy-dont la personnalité est seulement évoquée dans la thèse de théologie ${ }^{17}$. Notamment, dans son travail de 1950, Poulat avait suivi Blondel dans sa "Recherche d'une vision intégrale » (le titre du chapitre VII), qui aurait débouché sur le plan social en un modèle maritainien de chrétienté profane, où « chaque secteur de l'activité humaine sera défini dans sa parfaite autonomie et dans son intégrale relation à Dieu » (Poulat, 2015 : 280). Opportunément, l'éditeur de la thèse de 1950 (une année aussi significative dans l'histoire du pontificat de Pie XII et, plus généralement, de celle de la théologie catholique contemporaine) n'a pas manqué d'évoquer la

10. À ce sujet voir (Poulat, 2006b).

11. Il faut rappeler qu'Émile Poulat fut l'un des signataires du communiqué dit « Manifeste des 73 » publié dans L'Humanité du 3 février 1954.

12. Nous entendons donc confirmer et renforcer les thèses soutenues dans (Cavalin, 2009).

13. Voir (Suhard, 1947).

14. (Poulat, $2015: 198$ note 30 ).

15. Fr. Trémolières, "Introduction » (Poulat, 2015 : 30). D'ailleurs, dans la thèse de 1962, Poulat met Blondel à l'écart du modernisme, le rangeant plutôt parmi les «novateurs » (Poulat, 1996a : 21), et son jugement n'a pas trop bougé par la suite (voir ibid., p. XLV).

16. D'après la formule de Friedrich Heiler : (Poulat, 1996a : 7).

17. Émile Poulat, dans «Au terme du parcours. Les eaux mêlées de la recherche et de l'histoire (1954-1979-...) », a rappelé que lorsqu'il commença de travailler sur le modernisme Loisy lui était « un nom entendu », mais dont il n’avait rien lu (Poulat, $1982: 290$ ). 
«nuit critique » vécue en cette même année par Poulat ${ }^{18}$. À cette même époque, par sa correspondance suivie avec Mgr Joseph Veuillot, alors attaché à la Secrétairie d'État, Poulat put aussi se rendre compte que l'expérience des prêtresouvriers qu'il endossait soulevait des méfiances à Rome : elle était soupçonnée de cacher l'une des dangereuses formes de " néo-modernisme » que les autorités vaticanes étaient en train de pourfendre en France ${ }^{19}$.

L'intérêt que la crise moderniste avait soulevé chez le jeune clerc à la vocation théologique - entretenu par la publication que les jésuites blondéliens (très appréciés par le doctorant de Fribourg), notamment Henri de Lubac, faisaient des correspondances de Maurice Blondel ${ }^{20}$, ensuite renforcé par la tentative maladroite des autorités vaticanes, en 1958, de conditionner lourdement la vie du jeune Groupe de sociologie des religions ${ }^{21}$ - et un flair tout personnel de rat d'archives, affuté par la fréquentation des témoins et même des acteurs qui avaient survécu aux années tempétueuses de la crise moderniste (Giovanni Pioli, Joseph de Tonquédec, Mmes Nourry, Sartiaux et Louise Juston Sabatier, Jean Calvet et Albert Michel), poussèrent Poulat à sortir de l'oubli la biographie de Loisy qu'avaient préparée Houtin et son disciple Sartiaux ${ }^{22}$. Son édition critique de ce texte resté jusqu'alors dactylographié le conduisit à ressusciter un monde, en centrant la réflexion sur "l'énigme Loisy ", ce qui demeurera un axe portant de son œuvre jusqu'à l'ouvrage de 1984 qui vient clore l'enquête sur la crise moderniste, Critique et mystique, significativement sous-titré " Autour de Loisy » ${ }^{23}$. La proximité féconde en travaux avec Henri Desroche ${ }^{24}$, «compagnon

18. Fr. Trémolières, «Introduction" (Poulat, $2015: 36)$.

19. Voir (Poulat, 1986), notamment p. 303. "Sous Pie XII, le mot modernisme était dans toutes les têtes et sur beaucoup de lèvres" (Poulat, 1996a: XXXV).

20. Voir (Poulat, 1996a: 7).

21. L'orientation aconfessionnelle des recherches menées, impulsée par Le Bras et Desroche, allait placer les membres du groupe "au cœur de la "crise moderniste", nouvel épisode ", car le nonce apostolique Paolo Marella «pria G. Le Bras en 1958 de supprimer les Archives », sans évidemment pouvoir aboutir dans cette démarche (Poulat, 2006a : 33). On peut se demander jusqu'à quel point l'expérience de l'incident de 1958 a déteint sur l'évocation du modernisme tel qu'il l'avait «connu et vécu » que le doyen Le Bras fit avec "sa vivacité et son humeur habituels ", lors de la soutenance de thèse d'Émile Poulat en 1962 : voir (Bédarida, 1963 : 265).

22. Poulat a évoqué le déroulement de ses premières études sur la crise moderniste : "J'ai entamé le morceau par les Mémoires de Loisy. Deux milles pages serrées in-octavo : j’eus le sentiment que tout avait été dit. J'ai continué par l'Histoire de Rivière et la Vie de Loisy dont Houtin avait laissé le manuscrit : chacune présentait les choses à sa manière, leur donnait l'éclairage convenable à sa thèse, sévère pour Loisy. C'étaient sans doute, avec les dates et les références, leur seul point d'accord [...]. Ce n'était qu'un début : ces trois versions contradictoires des mêmes événements en laissaient présager d'autres à mesure que l'enquête [...] s'étendrait. Le problème n'était donc pas de savoir ce qui s'était réellement passé, selon la vielle formule de Ranke, dans les faits [...]. Il était de savoir ce qui s'était passé dans les esprits, dans la tête des acteurs. Les secrets des consciences, qui, heureusement, se laissent suivre à la trace " (Poulat, $1982:$ 292-293).

23. (Poulat, 1984).

24. Voir la «Bibliographie d'Émile Poulat (1950-2014)» éditée par Y. Tranvouez dans (Poulat, 2015) : numéros 4 et 5 (1956), 6 (1957) et 25 (1962), pour rester dans les limites temporelles de notre étude. 
et maître ${ }^{25}$, grand spécialiste des millénarismes contemporains, qui avait aussi été le référent de l'aile missionnaire ouvrière plus avancée d'Économie et humanisme, visé en 1950 par les censeurs du Saint-Office pour ses études sur Marx et le marxisme, est par ailleurs un autre élément à considérer du milieu intellectuel porteur pour pouvoir évaluer le parcours qui déboucha sur la thèse magistrale de 1962 centrée sur Loisy, dirigée par Gouhier et discutée avec Le Bras, Marrou et Dupront.

Néanmoins, entre la thèse de 1950 et celle de 1962, en passant du sillage de Blondel à celui de Loisy, intervinrent la crise de prêtres-ouvriers de 1954 et le choix pour Poulat de se ranger parmi les insoumis. Il n'a pas été très disert sur les circonstances personnelles de sa participation à la Mission de Paris et ce choix de rejoindre le camp des réfractaires en $1954^{26}$. Par ailleurs, l'on sait qu'avant de produire une histoire officielle des débuts des prêtres-ouvriers (Poulat, 1965), il s'en fit aussi d'abord l'historien clandestin ${ }^{27}$. Cela, dans le but de présenter au grand public l'exposé des faits concernant les "saints volés en enfer ", à partir du constat que la crise en cours renvoyait à l'affrontement de longue durée du christianisme, "et plus généralement [des] religions traditionnelles, avec une civilisation scientifique et technicienne, elle-même déchirée par les contradictions et les luttes qu'engendre en elle la loi du profit souverain ${ }^{28}$.

Or Émile Poulat, à plusieurs reprises, a mis en relation la crise moderniste avec celle des prêtres-ouvriers ${ }^{29}$, et ce dès avant 1962 . D'ailleurs, un simple coup

25. Titre de l'hommage qu'il lui a consacré, voir (Poulat, 1997).

26. À ce sujet voir les précieuses indications contenues dans l'entretien donné à Yvon Tranvouez et François Trémolières, publié comme «Postface. La théologie face à l'histoire. Retour sur un itinéraire " (Poulat, $2015: 287-296$ ).

27. Voir (Poulat, 1986), notamment p. 296-297, et A. Simonin, Les Éditions de Minuit, 1942-1955. Le devoir d'insoumission, Paris, IMEC, 1994, p. 437-443. Notons que, dans sa production "modernistica ", Émile Poulat a révélé une passion et une capacité remarquables dans l'étude critique de la littérature anonyme et pseudonyme. Il suffit de rappeler l'édition déjà citée du travail clandestin de Bremond sur Loisy, mais aussi l'annexe "Pseudonymes et anonymes modernistes " dans sa thèse de 1962. Et il est intéressant de noter que dans ce dernier texte, le recours à l'anonyme et au pseudonyme est présenté chez les modernistes surtout comme le «refus [...] de se laisser contraindre à l'alternative du silence ou du départ [...] dans le temps même où leur apparaissait comme un devoir de leur conscience et de leur sacerdoce de dire ce qu'ils pensaient et ce qu'ils ressentaient" (Poulat, 1996a : 647). Notamment chez Loisy, le recours au pseudonyme est présenté par Poulat comme une stratégie circonscrite et adoptée pour ne pas majorer les effets de publicité que le nom propre aurait produit et donc, pour ne pas provoquer ouvertement un scandale ecclésial (Poulat, 1996a : 644).

28. Selon l'introduction anonyme de l'ouvrage dont il s'était fait l'éditeur, Les Prêtresouvriers, Paris, Minuit, 1954, p. 17. Il est intéressant de noter que déjà dans sa thèse de 1950 Poulat avait signalé l'intensité et l'importance du choc entre le christianisme et la nouvelle société industrielle, mais sa perspective était plutôt ancrée alors à la théologie paulinienne de la lutte inexorable entre l'Église et le monde (voir Poulat, 2015:55 et 203) et cela selon une attitude que lui-même reconnaîtra plus tard comme caractéristique de l'intransigeance catholique (voir Poulat, $1977: 353$ ).

29. Voir par exemple (Poulat, 1986 : 302). D'après Poulat, si la crise des prêtres-ouvriers est une crise récente qui renvoie à une dynamique conflictuelle de longue durée (voir la note 
d'œil à sa bibliographie permet de comprendre que le livre de 1965 sur les prêtres-ouvriers a été en gestation simultanément à la thèse de 1962 sur la crise moderniste et nous avons déjà signalé comme l'expérience de compagnonnage avec les prêtres-ouvriers souleva des inquiétudes qui purent approfondir l'intérêt de Poulat pour la crise moderniste comme étant une crise ecclésiale non encore réglée. La rencontre en 1955 de l'insoumis (puis marié) avec Ignace Meyerson et la participation au Groupe de sociologie des religions qui s'en suivit ${ }^{30}$ permirent à Poulat de problématiser son expérience ecclésiale et de développer parallèlement ses recherches sur la crise moderniste, dont il rendra compte lors de ses participations régulières, entre 1956 et 1963, aux activités du Centre de psychologie comparative de Meyerson, où il présentera plusieurs exposés sur ses recherches en cours $^{31}$.

Le premier de ces exposés, donné le 4 décembre 1956, donna matière l'année suivante à un article dans le Journal de psychologie normale et pathologique d'Ignace Meyerson, intitulé « Notes sur la psychologie religieuse des prêtresouvriers » (Poulat, 1957). L'exposé souleva l'intérêt de Le Bras, qui avait jusqu'alors voulu dissuader Poulat de travailler sur les prêtres-ouvriers ${ }^{32}$. L'article de 1957 est un exercice de psychographie dynamique, effectué sur la base des données objectivées dans le texte anonyme de 1954 dont il avait été le maître d'œuvre - qu'il cite comme source, avec l'ouvrage inaugural des abbés Godin et Daniel, France, pays de mission? (1944). Cet article restera l'archétype de l'approche savante de l'expérience des prêtres ouvriers développée ensuite par Poulat (et même par ses épigones en la matière) ${ }^{33}$, mais nous allons voir que son importance ne s'arrête pas là.

ci-dessus), la crise moderniste, certes parfaitement situable dans le passé, garde quant à elle des effets importants dans l'actualité, car "au sein même des transformations qui se produisent, en effet, l'état des esprits garde une certaine constance tant que n'est pas aboli l'ordre des choses dont il procède et qui détermine d'insurmontables divisions" (Poulat, 1996a: 7-8).

30. Voir la "Préface» d'Émile Poulat à I. Meyerson, Existe-t-il une nature humaine? Psychologie historique, objective, comparative, Paris, Institut d'éd. Sanofi-Synthélabo, 2000, p. 7-13, notamment p. 8-9.

31. Poulat donna cinq exposés au Centre de Meyerson : un en 1956, deux en 1959, un en 1962 et un en 1963; deux sur des sujets relatifs aux prêtres au travail (1956 et 1959), deux sur des sujets liés à la crise moderniste (1959 et 1962) et un sur la question de l'approche historienne de la religion. Poulat intervint aussi avec une communication sur la notion de personne dans le catholicisme contemporain au colloque du Centre sur la personne en 1960. Voir sa contribution au volume d'hommage à I. Meyerson (Poulat, 1996b), spécialement p. 110-113 et 104 .

32. Voir (Poulat, 1996b : 101) et (Poulat, $2014: 67$ ).

33. Voir M. Guasco-Giuseppe Barra, Naissance des prêtres-ouvriers : Chiesa e mondo operaio. Le tappe d'un'evoluzione: da don Godin ai preti-operai, ai preti al lavoro, Turin, Gribaudi, 1967 ; Joseph Debès, Naissance de l'Action catholique ouvrière, Paris, Éditions ouvrières, 1982 ; Yvon Tranvouez, Catholiques et communistes. La crise du progressisme chrétien 19501955, Paris, Éditions du Cerf, 2000 ; et Marta Margotti, Preti e operai. La Mission de Paris dal 1953 al 1954, Turin, Paravia, 2000. 
D'après le jeune chercheur du GSR, au début de l'histoire des prêtres-ouvriers il y avait eu l'enthousiasme suscité chez certains membres du clergé par la lecture de France, pays de mission? La conscience avait jailli que l'Église n'était pas " en état de remplir sa mission religieuse à l'égard du prolétariat, en raison de la mentalité bourgeoise dont le clergé et les fidèles sont prisonniers " : l' " universalisme de l'Évangile» se trouvait "contrecarré par l'embourgeoisement de la prédication et de la vie chrétienne ", et si « la raison d'être de la foi et de l'Église » s'en trouvait atteinte, leur "substance " restait toutefois "intègre " (Poulat, 1957 : 53-54). En effet, « l'Église universelle » était « victime non d'une dégradation religieuse, mais d'un particularisme social », d'où son appel non pas « d'une religion temporalisée et trop humaine à une religion plus intérieure, mais de son accaparement par un groupe social [...] à une religion capable de parler aux simples ». Or, la voie indiquée par les abbés Godin et Daniel, celle du "passage de cette religion monopolisée à une religion "pure" ", passait non par le " retour aux sources écrites de la foi ", mais plutôt par le "dynamisme collectif de la communauté chrétienne ». Le projet d'une reconquête intégrale de la société et de la lutte contre l'embourgeoisement ecclésial avait été le caractère propre à la perspective ouverte par l'abbé Godin qui enthousiasma le clergé et les séminaristes. Avec Godin et Daniel, par conséquent, d'après Poulat, l'intransigeance s'ouvrait à l'intégralisme missionnaire, car, précisait-il :

L'abbé Godin et ses amis ne sont [donc] pas des réformateurs. Leur attitude novatrice procède directement de ce concept d'adaptation reçu du milieu ecclésiastique qui les a formés. Réforme et adaptation connotent également un appel à une transformation de l'Église, mais le premier l'invite à se régler sur son passé par un retour sur ellemême, l'autre à se tourner vers le présent en tant qu'il se passe hors d'elle. Au nom de la réforme, on invoque contre la tradition dominante une tradition plus ancienne, et si possible primitive; au nom de l'adaptation, on introduit un cadre de référence non théologique, dont les éléments, profanes, prêtent à la conscience religieuse un concours jugé indispensable et non plus suspect (Poulat, 1957 : 54).

En interprétant : la voie ouverte par les missionnaires était une voie distincte, certes novatrice, mais parallèle à celle de la "vraie réforme " ${ }^{34}$ du ressourcement. Pouvant s'autoriser de la première épître aux Corinthiens (1 Cor 9, 22), la voie indiquée était celle de l' "adaptation » au monde. Or, dans la suite du passage qu'on vient de citer, Poulat annonçait que de "graves questions » suivraient, car les «conduites d'adaptation» recelaient « des puissances redoutables qui refusent périodiquement de se laisser neutraliser, ainsi qu'il est apparu dans le cas des prêtres-ouvriers ».

34. Selon le titre de l'ouvrage du P. Congar, Vraie et fausse réforme dans l'Église (Éditions du Cerf, 1950), dont Poulat recensa en 1969 la réédition de 1968 dans les Archives de sociologie des religions (t. 28, p. 189-190). Dans cette recension, il exprime le souhait qu'Y. Congar puisse revoir son jugement sur les modernistes (comme il venait de le faire au sujet du protestantisme) et salue l'exigence que le dominicain venait de manifester de dépasser le projet d'une adaptation du christianisme au monde entendue en sens unique, comme adaptation du monde aux vérités chrétiennes. 
L'espace fait défaut pour pouvoir documenter les passages pathétiques où Poulat présenta l'authenticité du témoignage offert par les prêtres-ouvriers «au cœur du drame humain » qu'ils vécurent (Poulat, 1957 : 56-57). Ce qui nous intéresse ici c'est de voir avec lui que la voie du renouvellement missionnaire, née d'une culture religieuse intransigeante, devait conduire à l'alternative entre l' "intégration de l'incroyant dans un complexe socio-religieux qui s'impose par sa “supériorité" » et la "symbiose » par "naturalisation»: la voie propre des prêtres-ouvriers, où "à l'aventure exotique se substitue l'aventure spirituelle ", car « la conscience d'être porteur d'une vérité absolue, sans s'estomper, doit s'ouvrir à une préoccupation urgente beaucoup plus délicate : avant de parler il faut "en être" " et dans ce cas, continuait Poulat, "l'adaptation cesse d'être un ravalement de façade pour devenir une transformation intérieure " ${ }^{35}$. L'analogie est flagrante avec l'alternative sur le plan culturel entre les "novateurs ", comme Lagrange et Battifol, adversaires du modernisme, et le moderniste Loisy ${ }^{36}$, et plus en général avec les effets produits par les dynamiques de l'affrontement entre l'ordre intellectuel catholique (la science catholique) et les nouveaux savoirs profanes des sciences religieuses :

Face à la culture laïque, il [le renouveau intellectuel sous Léon XIII] se place en concurrence, avec le sentiment de sa supériorité intrinsèque [...]. Dans cette foulée, l'Université catholique de Louvain ouvrira une voie originale (Mgr Mercier [...]; Mgr Deploige), en cherchant à élaborer une "néo-scolastique " nourrie des progrès des sciences physiques, psychologiques et sociales. À la concurrence se substitue ainsi l'utilisation. Il apparut pourtant qu'un pas de plus était nécessaire, sinon deux : il ne suffisait pas d'assimiler des techniques et des résultats; il fallait en outre que le savant participe lui-même à un nouvel état d'esprit. Tout le débat entre catholiques convaincus de cette nécessité portera sur les exigences de cette véritable conversion intellectuelle.

Et plusieurs " tendances majeures ", continue le passage qu'on vient de citer, se laisseront discerner : les progressistes se persuaderont qu'elle [la véritable conversion intellectuelle] se limite à la maitrise d'une discipline, sans mettre en cause l'édifice théologique (Mgr Batiffol, le P. Lagrange, le P. de Grandmaison); les modernistes penseront qu'elle impose une révision profonde des idées reçues et, corrélativement du « régime intellectuel » dans l’Église (Loisy, Le Roy) ${ }^{37}$.

La voie empruntée par les prêtres-ouvriers, soutint Poulat dans son article de 1957, avait été tracée pour la première fois quand «la foi nouvelle, née en milieu juif, rencontra la civilisation hellénistique»(Poulat, 1957 : 58). C'était une voie faisant penser aux spirituels du Moyen Âge et qui dépassait celle des

35. (Poulat, 1957 : 58). En 1976, Poulat affirmera : « Le conflit entre l'Église et la bourgeoisie a engendré deux types rivaux de catholicisme, également soucieux d'une réforme religieuse " (Poulat, 1982: 101).

36. (Poulat, 1996a : 21).

37. Les deux extraits sont tirés d'un article de 1969 proposé aussi pour un recueil en italien et pour un article de l'Encyclopaedia Universalis, publié enfin dans É. Poulat, Modernistica. Horizons, Physionomies, débats, op. cit., p. 38-39. 
abbés démocrates, car "il n'était pas question d'aller au peuple : il fallait être peuple » (ibid.). L'entrée à l'usine aurait été le pas ultérieur franchi, comportant la "prolétarisation ", le denuo nasci accompagné d' " une identification matérielle et une conscience nouvelle» (Poulat, 1957:60). Notamment, sans « remettre en cause la substance du sacerdoce catholique tel que le définit la théologie traditionnelle ", les prêtres-ouvriers " à des degrés divers, et certains très profondément, en ont tous contesté la structure historique, qu'ils jugeaient incompatible avec la réalité de leur vie quotidienne ou [...] qu'ils voyaient comme l'expression d'un monde fini » et ils se sont pour cela "heurtés au désaveu formel de la hiérarchie catholique »(Poulat, 1957 : 61). Également sans mettre en discussion l'origine et la mission divine de l'Église, les prêtres-ouvriers avaient critiqué son inféodation à la société bourgeoise et refusé avec le peuple la foi selon sa représentation ecclésiale comme une forme d'aliénation, d'où la nécessaire intervention de l'autorité et la «crise » qui s'en suivit (Poulat, 1957 : 63-64). Et à nouveau une alternative se posa entre la soumission et l'insoumission. Et cette dernière fut en réalité une fidélité profonde à la vocation missionnaire initiale et à la vie nouvelle qui en était dérivée (Poulat, 1957: 65). Ainsi « se soumettre sans renier, c'était céder pour être entendu. Résister, c'était choisir d'être une écharde, un "problème vivant" dans la chair de l'Église ». Finalement, conclut Poulat, l'expérience des prêtres-ouvriers pouvait être considérée comme une expérience évangélique ou téméraire, cela selon le point de vue tenu ${ }^{38}$.

Comme celles des modernistes, les voies empruntées par les prêtres-ouvriers avaient donc été multiples et divergentes ${ }^{39}$. Et si Loisy n'a pas eu la chance d'être toléré dans l'Église de son temps comme un insoumis de 1954, même le moderniste par antonomase fut "un clerc qui n'a pas trahi ». Car, savant et religieux en même temps, il n'alla pas « de l'idéal chrétien à l'idéal éclairé, de la foi catholique à la foi positiviste. Il n'a pas comme d'autres contemporains, perdu la foi pour trouver la raison : telle une chrysalide, il a franchi un stade, "de la croyance à la foi”, a-t-il dit lui-même » ${ }^{40}$.

L'attention de deux historiens du mouvement missionnaire en France a été polarisée par un autre texte de 1957, un bulletin bibliographique publié dans Critique ${ }^{41}$, contenant le sévère compte rendu des deux volumes de l'Histoire

38. (Poulat, $1957: 66$ ). Voir la conclusion sur le sens du modernisme, également ouverte, dans (Poulat, 1996a : 620). Poulat a rappelé que sa thèse de 1962 fut reçue comme une écharde par un censeur de l'archevêché de Paris, qui avait flairé du subjectivisme dans les conclusions de l'ouvrage et reprocha à Casterman de ne pas avoir sollicité l'imprimatur (Poulat, 1996a : XII).

39. Multiplicité et divergence ont été aussi un acquis important des études de Poulat sur les modernistes, auquel il tenait beaucoup et opposant selon lui l'approche historienne à l'approche théologique conditionnée par la condamnation du Magistère : "Jamais le modernisme n'a constitué un mouvement homogène avec une pensée systématisée » (Poulat, 1996a : 9).

40. É. Poulat, «Avant-propos » (Poulat, 1984 : 9).

41. "Religion et politique. De l'abbé Grégoire aux prêtres-ouvriers ", dans Critique, 123124, août-septembre 1957, p. 757-770. Voir (Cavalin, 2009). 
religieuse de la France contemporaine d'Adrien Dansette et de celui que ce même auteur avait rédigé comme suite à son diptyque ${ }^{42}$, centré sur l'analyse de l'expérience des prêtres-ouvriers, où il avait critiqué l'intransigeance romaine, mais aussi les prêtres-ouvriers (et notamment les insoumis), traités d'excités en proie à un complexe de culpabilité à l'égard du prolétariat. Poulat a lui-même attiré l'attention sur ce texte de jeunesse en le rééditant dans un chapitre d'Église contre bourgeoisie, le maître ouvrage qui contient sa thèse sur le conflit triangulaire et l'importance matricielle du catholicisme intransigeant. La manière dont il le présente (Poulat, 1977: 64) mérite d'être analysée attentivement pour les affirmations qu'elle contient et pour la syntaxe abrupte de la phrase centrale qui demande un effort d'interprétation. En se référant vingt ans après à son compte rendu des trois volumes de Dansette, Poulat écrit :

J'y ai retrouvé avec étonnement et plaisir un fond d'idées qui ne se sont imposées à moi que lentement. Elles n'ont jamais été, en effet, l'hypothèse de travail qui a nourri mes premières recherches et que je me suis efforcé de valider. Tout au contraire, j'ai longtemps suspecté toute problématique reçue en ce domaine et leur force de suggestion me paraissait trop grande pour que je croie échapper à leur attraction simplement en refusant leur conformisme. C'est dans ces dispositions que j'ai commencé à reprendre de première main l'examen des données parfois déjà exploitées. Mes certitudes, mon assurance ne se sont établies que lentement et je ne suis parvenu que laborieusement à une compréhension qui me paraît s'imposer en rigueur de méthode.

Tout en rejetant l'hypothèse d'avoir produit des recherches téléguidées par des présupposés théoriques préalablement arrêtés (fidèle en cela à la leçon bachelardienne), dans ce passage Poulat avoue toutefois qu' " un fond d'idées » s'est imposé lentement dans son œuvre. Surtout, il avoue que les idées en question exerçaient déjà à cette époque une "force de suggestion » et une " attraction " tellement fortes sur son esprit qu'il a voulu les soumettre à l'épreuve de la critique documentaire et cela afin d'éviter de tomber trop naïvement sous leur emprise. En effet, dans le compte rendu de 1957 des livres de Dansette on retrouve certaines thèses sur lesquelles Poulat reviendra en 1977 (la commune matrice intransigeante du pape rénovateur Léon XIII et de son successeur intégriste, le refus d'une histoire religieuse du catholicisme limitée aux conflits intra-ecclésiaux, prétendument libre d'a priori politiques, mais l'expression en fait d'un catholicisme inféodé à l'ordre social bourgeois) ${ }^{43}$.

Si l'article produit pour la revue de Meyerson nous a permis de saisir la dynamique des mentalités annonçant le clivage des positionnements qui a servi

42. A. Dansette, Destin du catholicisme français (1926-1956), Paris, Flammarion, 1957.

43. Par ailleurs, un autre extrait du texte de la revue Critique de 1957 (p. 760-761), consistant dans une présentation cette fois-ci positive du roman Birama de Robert Delavignette, a été repris pratiquement à l'identique par Poulat dans la réédition de 1999 de son maître ouvrage de 1965 sur les prêtres-ouvriers, au moment de se congédier du lecteur et de se pencher sur les perspectives ouvertes par leur expérience et sur les effets ébranlants de leur exemple missionnaire dépassant le paradigme autoréférentiel de l'inculturation (Poulat, 1999 : 588-589). 
ensuite de grille interprétative pour une histoire du catholicisme dans son affrontement avec la démocratie bourgeoise et avec le socialisme, ce fut dans l'ample introduction à son édition critique des articles de l'abbé Calippe ${ }^{44}$ que Poulat traita explicitement de la question du travail des clercs d'après les modernistes. Dans ce texte, après avoir insisté sur le caractère éminemment intellectuel de l'œuvre entreprise par les modernistes, tout comme il l'avait fait en présentant les intentions des prêtres-ouvriers dans son article de 1957, Poulat tint à écarter l'idée que les modernistes eussent poursuivi un projet de réforme ecclésiale : "Ils rêvaient d'une Église renouvelée, ils n'avaient pas le tempérament de réformateurs et ils ne s'intéressèrent jamais, en France tout au moins, à un véritable plan de réforme ${ }^{45}$. "Après avoir ainsi écarté le risque éventuel que l'essai de renouvellement intellectuel constitué par le modernisme puisse représenter un exemple de "fausse réforme ", Poulat s'étendit sur le "développement inattendu » que le modernisme avait trouvé en Italie et, notamment, chez le disciple napolitain de Murri, Gennaro Avolio ${ }^{46}$. Ce dernier, dans ses positions extrêmes, avait prôné " un sacerdoce laïc dans la perspective d'un socialisme chrétien » (Poulat, 1961 : 137) pour un clergé au travail. L'expérience d'Avolio prit d'autant plus d'importance aux yeux de Poulat qu'elle était arrivée "à provoquer, à Rome même, une mise au point semi-officieuse d'un particulier intérêt » (Poulat, 1961 : 131) et cela de la part d'une personnalité très vraisemblablement déjà connue par le docteur de Fribourg ${ }^{47}$ et qui, par la suite, sera pour Poulat un objet privilégié de ses remarquables travaux sur l'intégrisme ${ }^{48}$, le "frère ennemi » du modernisme : Mgr Umberto Benigni. Car, dans sa Corrispondenza romana, l'infatigable polémiste avait attaqué Avolio et justement sur les mêmes points que Rome allait reprocher plus tard aux prêtres-ouvriers : la dénaturation du ministère sacerdotal aggravée par l'engagement partisan dans la mêlée des conflits sociaux ${ }^{49}$. Dans la suite de sa longue introduction aux articles de l'abbé Calippe, Poulat proposa à nouveau le schéma de l'origine intransigeante des options rivales que sont l'acculturation intégraliste et la naturalisation moderniste, à partir du souci missionnaire apparu au XIX e siècle, "celui de la Reconquête » :

44. (Poulat, 1961). Là encore, la parution de ce travail fut précédée en 1959 par un exposé donné à son sujet par Émile Poulat, au Centre de Meyerson.

45. (Poulat, 1961 : 128-129). Dans sa thèse de 1962, Poulat a repéré en Italie et notamment dans les positions d'Ernesto Buonaiuti le terrain où le modernisme vire à la "réforme religieuse " (Poulat, 1996a : 18 et n. 30).

46. Sur cette importante personnalité du modernisme italien, voir U. Parente, Riformismo religioso e sociale a Napoli tra Otto e Novecento. La figura e l'opera di Gennaro Avolio, Urbino, Quattroventi, 1995.

47. Voir (Poulat, $2015: 265$ ).

48. Outre l'ouvrage déjà mentionné (Poulat, 1977), signalons (Poulat, 1969) - encore un travail pionnier - et l'introduction de Poulat à son édition en fac-similé de La Corrispondenza romana : bulletin d'informations et notes. La Correspondance de Rome. Informations, documents, notes: Roma 1907-1912, trois volumes, Milan, Feltrinelli, 1971.

49. Voir (Poulat, $1961: 134$ ). 
La Révolution a renversé la situation de l'Église. Devant ce problème inédit vont s'affronter [...] deux grandes familles d'esprits, chacune proposant son interprétation de l'histoire et sa conception de la société chrétienne. Pour les uns, une partie du monde chrétien a fait sécession et s'est retournée contre l'Église; l'objectif essentiel est de restaurer l'État chrétien, grâce à une armature et à des cadres que seule l'Église peut lui fournir. Les autres voient naître hors de l'Église un monde nouveau dont les chrétiens ont à être le ferment, le levain; la tâche difficile est de refaire un peuple chrétien, d'irriguer avec le courant chrétien des terres nouvelles ${ }^{50}$.

Finalement, observa Poulat, si dans ses propositions l'abbé Calippe n'a pas dépassé le modèle intégral, d'autres développeront plus tard l'idée de mission en faisant "place à l'exigence d'animation d'une société sécularisée qu'on ne peut pénétrer qu'en se laissant d'abord pénétrer par elle ", laissant tomber des éléments autrement considérés comme fondamentaux de l'ordre catholique de la société (Poulat, 1961 : 172-173). L'allusion était évidente au programme des prêtres-ouvriers, mais aussi, sur le plan intellectuel, à la diaspora moderniste.

La crise des prêtres-ouvriers, qui fut aussi un drame personnel pour Poulat, possède une importance non négligeable pour comprendre le sens de la recherche sur le modernisme et sur l'histoire du catholicisme contemporain que mena l'insoumis tout au long de sa carrière. Et cela non seulement parce que la crise de 1954 délivra définitivement une vocation intellectuelle déjà esquissée, mais aussi parce qu'elle permit l'élaboration des catégories qui orienteront les recherches effectuées en obéissant à cette même vocation. L'expérience de la crise de 1954 fut en effet vécue et comprise par Poulat comme un effet révélateur d'une crise de longue durée qui reproduisait les mêmes dynamiques que la crise moderniste du début du siècle. Poulat élabora donc sa grille interprétative de l'histoire du catholicisme postrévolutionnaire à partir de sa compréhension du mouvement des prêtres-ouvriers, qu'il avait lui-même côtoyé. Il commença, notamment, à mettre en place les concepts antonymes qui rendent opératoire la grille en question : intransigeance/rénovation (intégration - acculturation - vraie réforme/adaptation - naturalisation). L'expérience de la crise des prêtres-ouvriers a donc permis non pas la découverte de l'importance de la crise moderniste (c'était déjà une donnée acquise en 1950 par le docteur en théologie), mais celle des arcanes mêmes de cette crise, révélatrice de l'histoire très conflictuelle du catholicisme contemporain. Dans cette perspective prend aussi sens l'affirmation en 1980, selon laquelle :

50. (Poulat, $1961:$ 167). Par là le spécialiste des sciences religieuses donnait la réponse au jeune docteur en théologie qui, en 1950, ayant fait état du choc entre "deux mentalités ", celle de «l'intégrisme doctrinal » et celle blondélienne, avait affirmé : " Nous sommes devant deux prises de vue opposées sur la réalité : est-il possible de trouver la source de cette opposition ?" En 1950, Poulat avait poursuivi son discours en rédigeant un paragraphe sur « Augustinisme et aristotélisme» (Poulat, $2015: 266-267)$. 
Malgré tout l'intérêt que je lui portais, l'histoire du modernisme n'était ni le foyer unique ni l'axe principal de mes préoccupations. Et elle ne l'est jamais devenue. Elle représentait pour moi un nœud stratégique sur l'échiquier d'un ensemble de problèmes plus généraux, dans l'immense partie où se jouait le destin historique du christianisme au sein d'une société qui en était sortie ${ }^{51}$.

Et toujours d'un point de vue rétrospectif, dans un texte plus récent encore (Poulat, 1996 : LXXV), il a précisé quelle était son «hypothèse de travail » lorsqu'il s'attacha au modernisme :

Le catholicisme moderne et contemporain m'apparaissait comme un système en état de crises à répétition et en évolution sous l'effet de ces crises. Il convenait donc d'examiner de très près les tensions et les luttes dont il était le siège, les acteurs et les enjeux qui s'y déployaient, les contraintes et les ressources qui expliquaient à la fois le maintien du système, son incroyable résistance dans les tempêtes les plus agitées, son étonnante capacité d'adaptation à toutes circonstances.

On pourrait dire que l'étude de la crise moderniste, avec ses particularités par rapport à celles qui suivront, notamment par son envergure intellectuelle et sa mise en discussion radicale de l'orthodoxie, fut la loupe qui permit à Poulat de s'attacher à la crise des prêtres-ouvriers un demi-siècle plus tard et d'en retrouver le problème de fond, avec la nécessaire distance prise par rapport à un objet historique dont il portait les marques dans sa propre personne. Le rejet par Poulat de la naïveté positiviste n'a pas été la parure d'un sociologue avisé des questions d'épistémologie historique. Les critiques adressées par les historiens au style " froid » de l'auteur de la thèse de 1962 frappaient dans le vide. D'autres ont par la suite manifesté leurs insatisfactions sur l'étude des crises historiques du catholicisme contemporain à l'aune d'une grille sociologique par nature trop rigide. Néanmoins, reste la fécondité des recherches de Poulat consécutives à la crise de 1954. L'intérêt pour son approche de l'histoire du catholicisme a certainement pâti de l'infléchissement historiographique de l'histoire sociale vers l'histoire culturelle et sans doute cela n'a pas favorisé non plus le développement des études françaises sur la crise moderniste qui, comme l'avait remarqué Poulat, fut en France une crise qui toucha surtout un réseau restreint d'intellectuels. Mais ce sont là des problèmes qui ne relèvent plus de cette étude.

\section{Giacomo LOSITO \\ Archives Maurice Blondel \\ Université catholique de Louvain \\ lositogy@libero.it}

\footnotetext{
51. «Avant-Propos» (Poulat, 1982 : 7). Ainsi dans cette même perspective, chez Poulat, "le "modernisme" cesse d'être l'aventure solitaire de quelques clercs ou universitaire d'avantgarde : en fait, il est la secousse annonciatrice du grand ébranlement qui va gagner tout le corps social catholique et que nous voyons à l'œuvre depuis quelques années à ciel ouvert " (Poulat, 1982 : 30 ; il s'agit de la reprise d'un texte de 1976).
} 


\section{Bibliographie}

BÉDARIDA François, SAUZET Robert, 1963, "Soutenance de thèses pour le doctorat ès lettres soutenues devant les Facultés des Lettres et des Sciences humaines ", Revue historique, CCXXX-4, p. 262-267.

Cavalin Tangi, Viet-Depaule Nathalie, 2009, «Des prêtres-ouvriers au mouvement missionnaire français. Bilan historiographique et nouvelles perspectives ", Histoire et missions chrétiennes, 2009-1, p. 9-41.

Houtin Albert, Sartiaux Félix, 1960, Alfred Loisy. Sa vie, son ouvre, Paris, Éditions du CNRS.

Poulat Émile, 1957, " Notes sur la psychologie religieuse des prêtres-ouvriers ", Journal de psychologie normale et pathologique, 1957-1, p. 51-66.

-, 1958, Les "Semaines religieuses ». Approche socio-historique et bibliographique, polytypé, Paris, Fondation nationale de sciences politiques/Groupe de sociologie des religions.

-, 1961, Utopie ou Anticipation? Le "Journal d'un prêtre d'après-demain » (1902-1903) de l'abbé Calippe, préface de Gabriel Le Bras, Paris-Tournai, Casterman.

-, 1965, Naissance des Prêtres-ouvriers, Paris-Tournai, Casterman.

,- 1969 , Intégrisme et catholicisme intégral. Un réseau secret international antimoderniste, la "Sapinière » (1909-1912), Paris-Tournai, Casterman.

-, 1972, Une cuvre clandestine d'Henri Bremond. Sylvain Leblanc. "Un clerc qui n'a pas trahi. Alfred Loisy d'après ses Mémoires" (1931), Rome, Edizioni di storia e letteratura.

-, 1977, Catholicisme, démocratie, socialisme : le mouvement catholique et Mgr Benigni, de la naissance du socialisme à la victoire du fascisme, Paris-Tournai, Casterman.

-, 1982, Modernistica. Horizons, physionomies, débats, Paris, Nouvelles Éditions latines.

-, 1984, Critique et mystique. Autour de Loisy ou la conscience catholique et l'esprit moderne, Paris, Le Centurion.

-, 1986, "Chiesa e mondo moderno : il caso dei preti operai ", in Riccardi A. (éd.), Pio XII, Bari-Rome, Laterza, p. 295-306.

-, 1996a, Histoire, dogme et critique dans la crise moderniste, $3^{\mathrm{e}}$ édition, Albin Michel (1962 pour la $1^{\text {re }}$ édition, Paris-Tournai, Casterman).

-, 1996b, "Le centre de psychologie contemporaine et les catholiques ", in Parot F. (éd.), Pour une psychologie historique. En hommage à Ignace Meyerson; Paris, PUF, p. 98118.

-, 1997, «Henri Desroche, compagnon et maître ", in Poulat É., Ravelet C. (éds.), Henri Desroche : un passeur de frontières. Hommages, Paris, L'Harmattan, p. 9-13.

-, 1999, Les Prêtres-ouvriers. Naissance et fin, Paris, Éditions du Cerf.

-, 2006a, Église contre bourgeoisie : introduction au devenir du catholicisme actuel, $2^{\mathrm{e}}$ édition, Paris, Berg international (1977 pour la $1^{\text {re }}$ édition, Paris-Tournai, Casterman).

-, 2006b, "Aux origines du "Groupe de Sociologie des Religions" ", Archives des sciences sociales des religions, 2006-4, p. 25-37.

-, 2014, L'Histoire savante devant le fait chrétien, Paris, Parole et Silence/Collège des Bernardins.

-, 2015, Le désir de voir Dieu et sa signification pour la théologie française contemporaine, introduction et édition du texte par François Trémolières, bibliographie par Yvon Tranvouez, suivi d'un entretien de l'auteur avec Y. Tranvouez et F. Trémolières, Paris, Desclée de Brouwer.

SuHARd Célestin Emmanuel, 1947, Essor ou déclin de l'Église : lettre pastorale. Carême de l'an de grâce 1947, Paris, Les éditions du Vitrail. 